\title{
Quantum Simulation of the Ultrastrong-Coupling Dynamics in Circuit Quantum Electrodynamics
}

\author{
D. Ballester, ${ }^{1}$ G. Romero, ${ }^{1}$ J. J. García-Ripoll, ${ }^{2}$ F. Deppe, ${ }^{3,4}$ and E. Solano ${ }^{1,5}$ \\ ${ }^{1}$ Departamento de Química Física, Universidad del País Vasco UPV/EHU, Apartado 644, 48080 Bilbao, Spain \\ ${ }^{2}$ Instituto de Física Fundamental, CSIC, Serrano 113-bis, 28006 Madrid, Spain \\ ${ }^{3}$ Walther-Meißner-Institut, Bayerische Akademie der Wissenschaften, D-85748 Garching, Germany \\ ${ }^{4}$ Physik-Department, Technische Universität München, D-85748 Garching, Germany \\ ${ }^{5}$ IKERBASQUE, Basque Foundation for Science, Alameda Urquijo 36, 48011 Bilbao, Spain \\ (Received 9 August 2011; revised manuscript received 16 March 2012; published 16 May 2012)
}

\begin{abstract}
We propose a method to get experimental access to the physics of the ultrastrong- and deep-strongcoupling regimes of light-matter interaction through the quantum simulation of their dynamics in standard circuit QED. The method makes use of a two-tone driving scheme, using state-of-the-art circuit-QED technology, and can be easily extended to general cavity-QED setups. We provide examples of ultrastrong- and deep-strong-coupling quantum effects that would be otherwise inaccessible.
\end{abstract}

DOI: 10.1103/PhysRevX.2.021007

\section{INTRODUCTION}

The Jaynes-Cummings model (JCM) [1] is a cornerstone of the field of quantum optics. It describes the interaction between a quantized electromagnetic (EM) field mode and a two-level atom under two important assumptions. First, the interaction is accurately modeled by a dipolar coupling between the field and the atom. Second, one can apply the rotating-wave approximation (RWA) because the coupling is small enough when compared to the sum of the frequencies of the two-level atom and EM field. These restrictions yield a solvable model where the atom and field exchange one excitation.

To study experimentally the physics of the JCM, the interaction needs to reach the strong-coupling (SC) regime. This can be done by isolating the two-level system from free space by means of a cavity with highly reflecting mirrors, making the coupling strength much larger than the spontaneous emission rate and the cavity decay rate. This type of setup is known as cavity quantum electrodynamics (cavity QED) [2,3]. Many relevant features of the JCM have been successfully tested in actual experiments using cavity-QED technology. For instance, the observation of the vacuum Rabi mode splitting in the optical domain with alkali atoms was reported [4], while in the microwave regime, vacuum Rabi oscillations using Rydberg atoms have also been realized [3,5].

In 2004, an important step forward was made when an artificial two-level atom (or a qubit) was shown to be strongly coupled to the EM field inside a superconducting 1D transmission-line resonator [6]. The newly born circuitQED technology was rapidly recognized as a promising

Published by the American Physical Society under the terms of the Creative Commons Attribution 3.0 License. Further distribution of this work must maintain attribution to the author(s) and the published article's title, journal citation, and DOI.
Subject Areas: Quantum Physics, Quantum Information

architectural platform from which the study of light-matter interaction can be extended [7]. Although most circuit-QED implementations were restricted to the SC regime of the JCM, key experiments showing the breakdown of the RWA have been recently realized in semiconductor microcavities [8] and circuit QED $[9,10]$. They have opened up new directions of research into the ultrastrong-coupling (USC) regime of light-matter interaction $[11,12]$, where the RWA can no longer be used, leading to novel features such as the creation of photons from the quantum vacuum [11]. Even though these works show that reaching the ultrafast dynamics is feasible, its controllability becomes very demanding as the light-matter coupling increases [13].

In this work, we introduce the quantum simulation of the USC and deep-strong-coupling (DSC) dynamics in circuit QED with a qubit-cavity system in the SC regime. Our treatment makes use of a novel two-tone orthogonal driving to the qubit. We show through analytical and numerical calculations that our proposal will have access to the regimes of USC $(0.1 \lesssim g / \omega \lesssim 1$, where $g / \omega$ is the ratio of the coupling over the resonator frequency) and DSC [14] $(g / \omega \gtrsim 1)$. This will pave the way for the implementation of a quantum simulator [15] for a wide range of regimes of light-matter coupling [16] in systems where they are unattainable. As we will discuss below, this includes the simulation of relativistic quantum phenomena, the Dicke spin-boson model, Kondo physics, and Jahn-Teller instability [17]. Although we present our method in the language of circuit QED, it can also be realized in microwave cavity QED $[3,18]$.

\section{THE MODEL}

The physical system we consider consists of a superconducting qubit strongly coupled to a microwaveresonator mode. If we work in the qubit degeneracy point, the Hamiltonian is given by [6] 


$$
\mathcal{H}=\frac{\hbar \omega_{q}}{2} \sigma_{z}+\hbar \omega a^{\dagger} a-\hbar g \sigma_{x}\left(a+a^{\dagger}\right),
$$

where $\omega_{q}, \omega$ are the qubit and photon frequencies, and $g$ stands for the coupling strength. Likewise $a\left(a^{\dagger}\right)$ represent the annihilation (creation) operators of the photon-field mode, whereas $\sigma_{x}=\sigma^{+}+\sigma=|e\rangle\langle g|+| g\rangle\langle e|, \quad \sigma_{z}=$ $|e\rangle\langle e|-| g\rangle\langle g|$, where $|g\rangle,|e\rangle$ are the ground and excited eigenstates of the qubit. In a typical circuit-QED implementation, this Hamiltonian can be simplified further by applying the RWA. Precisely, if $\left\{\left|\omega-\omega_{q}\right|, g\right\} \ll \omega+\omega_{q}$ [19], then it can be effectively approximated as

$$
\mathcal{H}=\frac{\hbar \omega_{q}}{2} \sigma_{z}+\hbar \omega a^{\dagger} a-\hbar g\left(\sigma^{\dagger} a+\sigma a^{\dagger}\right),
$$

which resembles the celebrated JCM of quantum optics. Performing the RWA amounts to neglecting counterrotating terms, $\sigma a$ and $\sigma^{+} a^{+}$, leading to a Hamiltonian equation, (2), where the number of excitations is conserved.

This Hamiltonian, (2), will be the starting point of our derivations. Consider that the qubit is now orthogonally driven by two classical fields. The Hamiltonian of the driven system reads

$$
\begin{aligned}
\mathcal{H}= & \frac{\hbar \omega_{q}}{2} \sigma_{z}+\hbar \omega a^{\dagger} a-\hbar g\left(\sigma^{\dagger} a+\sigma a^{\dagger}\right) \\
& -\hbar \Omega_{1}\left(e^{i \omega_{1} t} \sigma+e^{-i \omega_{1} t} \sigma^{\dagger}\right)-\hbar \Omega_{2}\left(e^{i \omega_{2} t} \sigma+e^{-i \omega_{2} t} \sigma^{\dagger}\right),
\end{aligned}
$$

where $\Omega_{j}, \omega_{j}$ stand for the amplitude and frequency of the $j$ th driving. Note that the orthogonal drivings couple to the qubit in a similar fashion as the resonator field. To obtain (3), we have implicitly assumed that the RWA applies not only to the qubit-resonator interaction term, but also to both drivings. Next, we will write (3) in the reference frame rotating with the frequency of the first driving, $\omega_{1}$, that is

$$
\begin{aligned}
\mathcal{H}^{L_{1}}= & \hbar \frac{\omega_{q}-\omega_{1}}{2} \sigma_{z}+\hbar\left(\omega-\omega_{1}\right) a^{\dagger} a-\hbar g\left(\sigma^{\dagger} a+\sigma a^{\dagger}\right) \\
& -\hbar \Omega_{1}\left(\sigma+\sigma^{\dagger}\right)-\hbar \Omega_{2}\left(e^{i\left(\omega_{2}-\omega_{1}\right) t} \sigma+e^{-i\left(\omega_{2}-\omega_{1}\right) t} \sigma^{\dagger}\right) .
\end{aligned}
$$

This allows us to transform the original first driving term into a time-independent one $\mathcal{H}_{0}^{L_{1}}=-\hbar \Omega_{1}\left(\sigma+\sigma^{\dagger}\right)$, leaving the excitation number unchanged. We will assume this to be the most significant term and treat the others perturbatively by going into the interaction picture with respect to $\mathcal{H}_{0}^{L_{1}}, \mathcal{H}^{I}(t)=e^{i \mathcal{H}_{0}^{L_{1}} t / \hbar}\left(\mathcal{H}^{L_{1}}-\mathcal{H}_{0}^{L_{1}}\right) e^{-i \mathcal{H}_{0}^{L_{1}} t / \hbar}$. The physical implementation of this transformation based on an additional drive pulse is described later in the text. Using the rotated spin basis $| \pm\rangle=(|g\rangle \pm|e\rangle) / \sqrt{2}$, we have

$$
\begin{aligned}
\mathcal{H}^{I}(t)= & -\hbar \frac{\omega_{q}-\omega_{1}}{2}\left(e^{-i 2 \Omega_{1} t}|+\rangle\langle-|+\text { H.c. }\right)+\hbar\left(\omega-\omega_{1}\right) a^{\dagger} a \\
& -\frac{\hbar g}{2}\left(\left\{|+\rangle\langle+|-|-\rangle\left\langle-\left|+e^{-i 2 \Omega_{1} t}\right|+\right\rangle\langle-|\right.\right. \\
& \left.\left.-e^{i 2 \Omega_{1} t}|-\rangle\langle+|\right\} a+\text { H.c. }\right) \\
& -\frac{\hbar \Omega_{2}}{2}\left(\left\{|+\rangle\langle+|-|-\rangle\left\langle-\left|-e^{-i 2 \Omega_{1} t}\right|+\right\rangle\langle-|\right.\right. \\
& \left.\left.+e^{i 2 \Omega_{1} t}|-\rangle\langle+|\right\} e^{i\left(\omega_{2}-\omega_{1}\right) t}+\text { H.c. }\right)
\end{aligned}
$$

By tuning the parameters of the external drivings as $\omega_{1}-$ $\omega_{2}=2 \Omega_{1}$, we can choose the resonant terms in this timedependent Hamiltonian. Then, if we have a relatively strong first driving, $\Omega_{1}$, we can approximate the expression above by a time-independent effective Hamiltonian as

$\mathcal{H}_{\text {eff }}=\hbar\left(\omega-\omega_{1}\right) a^{\dagger} a+\frac{\hbar \Omega_{2}}{2} \sigma_{z}-\frac{\hbar g}{2} \sigma_{x}\left(a+a^{\dagger}\right)$.

Note the resemblance between the original Hamiltonian (1) and (6). While the value of the coupling $g$ is fixed in (6), we can still tailor the effective parameters by tuning the amplitudes and frequencies of the drivings. If we can reach values such that $\Omega_{2} \sim\left(\omega-\omega_{1}\right) \sim g / 2$, the dynamics of the original system will simulate that of a qubit coupled to the resonator with a relative interaction strength beyond the SC regime-ideally in the USC and DSC. The coupling regime reproduced through the effective Hamiltonian (6) can be quantified by the ratio $g_{\text {eff }} / \omega_{\text {eff }}$, where $g_{\text {eff }} \equiv g / 2$ and $\omega_{\text {eff }} \equiv \omega-\omega_{1}$.

\section{NUMERICAL ANALYSIS}

To study the feasibility of our proposal, we have performed numerical calculations with realistic parameters for circuit QED [6] and compared the evolution described by the exact and effective Hamiltonians. In principle, the choice of $\Omega_{1}$ is unimportant as long as it is strong compared to the other parameters involved in this problem. Indeed, the evolutions of the Hamiltonians of Eqs. (3) and (6) become more similar with increasing $\Omega_{1}$. Nonetheless, experimental restrictions are expected to set the limit of this driving strength. Throughout the rest of this work, we assume $\Omega_{1} \sim 2 \pi \times 0.7 \mathrm{GHz}$, which is a realistic value.

After the discussion regarding the DSC dynamics [14], an interesting experiment would be the following. Assume we prepare the original SC undriven system in its ground state, i.e., $|g, 0\rangle$, and then at time $t=0$ we switch on the external drivings. Now the system evolves according to the unitary operator, which is computed by integrating the driven Hamiltonian equation, (3). For the sake of simplicity, we now assume that the evolution of the state is calculated in the rotating reference frame of Hamiltonian $\mathcal{H}^{I}(t)$. Later we will discuss how this step can be implemented. The solid line in Fig. 1 shows the evolution of $P_{g}(t)$ for two different cases. Both show a very good agreement when compared to the same probability but 

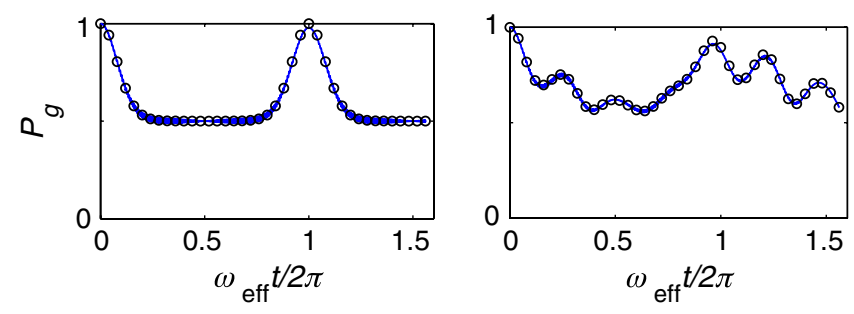

FIG. 1. $P_{g}(t)$ obtained by integrating the exact (solid line) equation, (3), and the effective (circles) Hamiltonian equation, (6). With the set of parameters in the text, $\Omega_{1}=2 \pi \times 700 \mathrm{MHz}$, we have two cases: left panel: $\Omega_{2}=0$, right panel: $\Omega_{2}=$ $2 \pi \times 10 \mathrm{MHz}$. The simulated ratio is $g_{\text {eff }} / \omega_{\text {eff }}=1$.

computed from the effective Hamiltonian (circles) of Eq. (6) derived in the strong driving limit. All these simulations are done with values of $\omega_{q}=2 \pi \times 8.01 \mathrm{GHz}, \omega=$ $2 \pi \times 8.01 \mathrm{GHz}, g=2 \pi \times 20 \mathrm{MHz}, \omega_{1}=2 \pi \times 8 \mathrm{GHz}$, $\omega_{2}=2 \pi \times 6.6 \mathrm{GHz}$, and $\Omega_{1}=2 \pi \times 0.7 \mathrm{GHz}$. Clearly such an amplitude for the first strong driving suffices, even to simulate the dynamics of a system reaching $g_{\text {eff }} / \omega_{\text {eff }}=1$. A feature characteristic of the DSC dynamics is the existence of a well-defined periodic evolution for the probability, $P_{g}(t)$, in the case of a degenerate qubit. Starting from $1, P_{g}(t)$ decays to 0.5 quite rapidly in order to have a subsequent revival at a time that is equal to the inverse of the effective resonator frequency. This is accompanied by the generation of photon-number wave packets that oscillate in time [14]. Putting $\Omega_{2}=0$ (Fig. 1, left panel) in our simulation, we observe that $P_{g}(t)$ presents nearly perfect revivals that take place at $\omega_{\text {eff }} t_{\text {rev }} \equiv$ $g_{\text {eff }} t_{\text {rev }}=2 \pi$, which corresponds to $t_{\text {rev }}=0.1 \mu \mathrm{sec}$ for the set of parameters used. If the effective energy of the simulated qubit $\Omega_{2}$ is not zero (Fig. 1, right panel) the evolution becomes nonperiodic, producing a distortion of the revival peak, which no longer reaches unity.

\section{ADDITIONAL DRIVE PULSE}

The computation of the probability $P_{g}(t)$ for the exact Hamiltonian equation, (3), in Fig. 1 has been done in the rotating reference frame used to derive Hamiltonian $\mathcal{H}^{I}(t)$. However, without going into this interaction picture, the evolution of $P_{g}(t)$ would show a fast oscillating term, depicted by the light solid line shown in Fig. 2. Here, we propose the following scheme based on an additional drive pulse, in order to get rid of this strong oscillation in an experiment. Imagine that after letting the system evolve with the Hamiltonian of Eq. (3) for a time $t$, we switch off nonadiabatically both of the external drivings and apply a detuning of about $-2 \pi \times 200 \mathrm{MHz}$ to the frequency of the qubit $\omega_{q}$ from its original value $2 \pi \times 8.01 \mathrm{GHz}$. Next, we switch on a third driving with the frequency detuned by $-2 \pi \times 200 \mathrm{MHz}$, from the value of $\omega_{1}=2 \pi \times 8 \mathrm{GHz}$, and with amplitude $-\Omega_{1}$ (opposite phase relative to the first one). The application of this additional drive pulse will
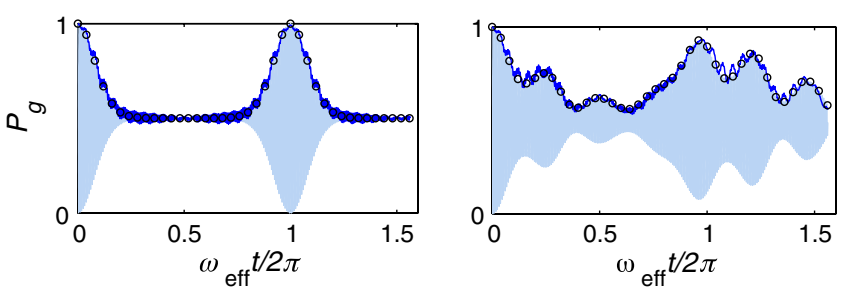

FIG. 2. $\quad P_{g}(t)$ obtained by integrating the exact Hamiltonian equation, (3), (light solid line, shaded area), and after applying an additional drive pulse (dark solid). Both are compared to the effective (circles) Hamiltonian equation, (6), evolution. The same parameters of Fig. 1 are used: left panel: $\Omega_{2}=0$, right panel: $\Omega_{2}=$ $2 \pi \times 10 \mathrm{MHz}$. During the additional drive pulse, qubit energy and driving frequency are detuned by $-2 \pi \times 200 \mathrm{MHz}$.

take the same time $t$. The wiggly dark solid line of Fig. 2 corresponds to the measurement of $P_{g}(t)$ following this method, which matches the evolution obtained from the effective (circles) Hamiltonian equation, (6).

\section{NONCLASSICAL STATES AND DIRAC EQUATION}

Nonclassical states of the EM field are a paramount resource for quantum information processing. However, their generation using all-optical devices is challenging due to the lack of strong nonlinearities. Through the strong coupling between a qubit and a confined EM field, circuitand cavity-QED technology provides a way to overcome these limitations [20-22]. Here we show that our setup can be used to generate highly nonclassical states of the field mode, without requiring the projective measurement of the qubit and/or the ability to control accurately the qubit-field interaction strength.

The nonclassicality of a bosonic field can be signaled by the Wigner quasiprobability distribution function (WF), which is defined as

$$
W(\alpha)=\frac{2}{\pi} \operatorname{Tr}\left[D^{\dagger}(\alpha) \rho_{f} D(\alpha)(-1)^{a^{\dagger} a}\right],
$$

where $\rho_{f}$ is the field-density matrix and $D(\alpha)=$ $\exp \left(\alpha a^{\dagger}-\alpha^{*} a\right)$ is the coherent displacement operator with amplitude $\alpha$. To show the ability of our setup to produce nonclassical states of the EM field, we plot in Fig. 3 the WF of the field for the same set of parameters studied before, with the the evolution time set at $g_{\text {eff }} t=\pi$. The top-left panel in Fig. 3 depicts the WF of the state generated when $\Omega_{2}=0$ and the qubit is measured in its ground state. The result is a well-known coherent Schrödinger-cat state with time-dependent amplitude $\left(g_{\text {eff }} / \omega_{\text {eff }}\right)\left(e^{-i \omega_{\text {eff }} t}-1\right)$, which is nonclassical. However, when the qubit is not measured, the state of the field after tracing out over the qubit will have as WF the plot of topright panel in Fig. 3, where a classical mixture of coherent states with opposite phases is left and any quantum feature is erased. When $\Omega_{2} \neq 0$, a surprising property shows up 

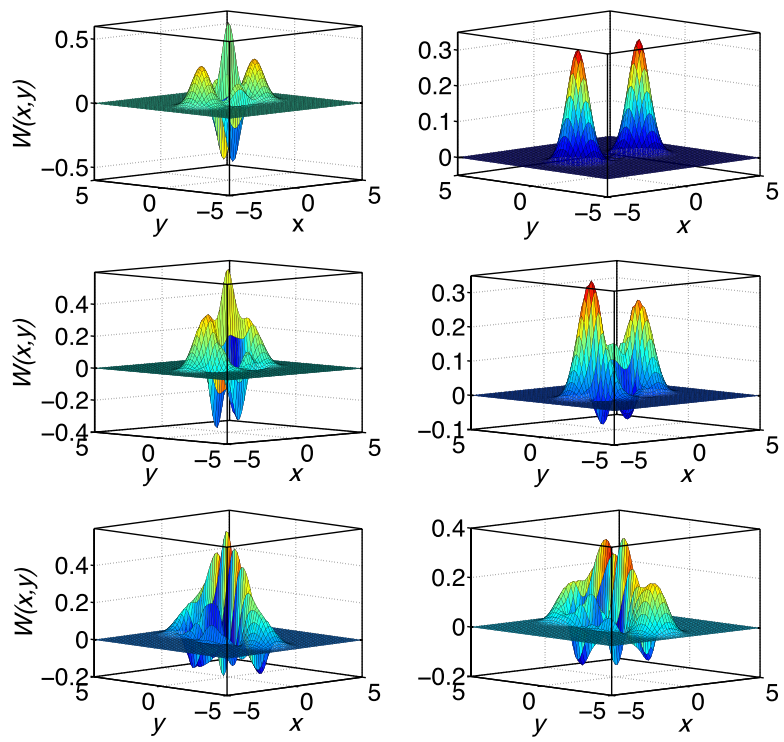

FIG. 3. Wigner function $W(x, y)$ of the field state after interaction time $g_{\text {eff }} t=\pi$, calculated $a b$ initio with the parameters of Fig. 1. Top-left panel: $\Omega_{2}=0, \omega_{\text {eff }}=g_{\text {eff }}$, qubit postselected in the ground state. Top-right panel: $\Omega_{2}=0, \omega_{\text {eff }}=g_{\text {eff }}$, qubit traced out. Center-left panel: $\Omega_{2}=2 \pi \times 10 \mathrm{MHz}$, $\omega_{\text {eff }}=g_{\text {eff }}$, qubit postselected in the ground state. Center-right panel: $\Omega_{2}=2 \pi \times 10 \mathrm{MHz}, \omega_{\text {eff }}=g_{\text {eff }}$, qubit traced out. Bottom-left panel: $\Omega_{2}=2 \pi \times 10 \mathrm{MHz}, \quad \omega_{\text {eff }}=0$, qubit postselected in the ground state. Bottom-right panel: $\Omega_{2}=$ $2 \pi \times 10 \mathrm{MHz}, \omega_{\text {eff }}=0$, qubit traced out.

after the qubit is traced out: The nonclassicality of the field is not completely lost (center-right panel in Fig. 3). This effect is more evident when $\omega_{\text {eff }}=0$ (bottom-right panel in Fig. 3). The setup might be enhanced by taking advantage of the cavity output and novel measurement techniques [23] to produce nonclassical propagating microwaves and lasing in circuit-QED [24].

The case of $\omega_{\text {eff }}=0$ is physically most relevant, as it relates to the quantum simulation of the $1+1$ Dirac equation [25]. If one repeats the derivation from Eq. (3) but assumes that both external driving come with an additional phase, $\phi=\pi / 2$, i.e., $-\hbar \Omega_{j}\left(e^{i\left(\omega_{j} t+\phi\right)} \sigma+\right.$ H.c. $)$, and uses the rotated qubit basis $\left| \pm_{\phi}\right\rangle=\left(|g\rangle \pm e^{-i \phi}|e\rangle\right) / \sqrt{2}$, then $\mathcal{H}_{\text {eff }}$ becomes

$$
\mathcal{H}_{\mathrm{D}}=\frac{\hbar \Omega_{2}}{2} \sigma_{z}+\frac{\hbar g}{\sqrt{2}} \sigma_{y} \hat{p},
$$

for $\omega=\omega_{1}, \sigma_{y}=i\left(\sigma-\sigma^{\dagger}\right), \hat{p}=-i\left(a-a^{\dagger}\right) / \sqrt{2}$. In the appropriate representation, the Schrödinger equation of our system is now formally equivalent to that of the $1+1$ Dirac equation of a relativistic spin-1/2 particle,

$$
i \hbar \frac{d \psi}{d t}=\left(c \hat{p} \sigma_{y}+m c^{2} \sigma_{z}\right) \psi,
$$

where $\hat{p}$ represents the momentum of a Dirac particle of mass $m$ and $c$ represents the speed of light. This shows the ability of our proposal to access a wide range of physical models. Through the engineered Hamiltonian, a qubit at rest coupled to the EM field would behave as a massive spin- $1 / 2$ particle moving near the speed of light. To observe peculiar effects, such as Zitterbewegung, in our setup one must pay attention at the mapping of the bosonic degree of freedom. While in the original Dirac equation, (9), the operator $\hat{p}$ corresponds to the momentum of the Dirac particle, in our Hamiltonian Eq. (8) this role is played by the quadrature $\hat{p}=-i\left(a-a^{\dagger}\right) / \sqrt{2}$ of the EM field mode in the resonator. Hence, in the simulated Dirac equation the dynamics of Zitterbewegung will show up in the expectation value of the field quadrature $\hat{x}=$ $\left(a+a^{\dagger}\right) / \sqrt{2}$, which has the same time evolution as the expectation value of the position operator $\hat{x}$ of a Dirac particle.

\section{DISCUSSION AND CONCLUSIONS}

Although we have disregarded the possible coupling between the orthogonal driving and the resonator field, it is easy to show that the effect of such a spurious coupling could be sorted out by adding a driving to the cavity (acting as a counter coherent displacement). Another source of error stems from qubit dephasing and relaxation rates, as well as the cavity decay rate. Nonetheless, the interaction times considered in all numerical simulations are well below standard decoherence times. To avoid the excitation of higher levels in the qubit, it is possible to design flux qubits where the splitting to the third level is above $30 \mathrm{GHz}[9,10]$. Our method can be extended to the case of a slightly anharmonic qubit via Gaussian shaped derivative removal by adiabatic-gate pulses [26].

The proposed quantum simulation of a broad range of regimes of light-matter coupling may become a building block in simulations of physics inaccessible in standard quantum optics. This includes the Dicke model for multiple qubits, the spin-boson model when the resonator is replaced by an open transmission line, Jahn-Teller instability for several discrete modes in the resonator, and relativistic quantum physics such as the scattering of relativistic particles [27]. Efficient computations of these problems may be beyond current numerical capabilities.

\section{ACKNOWLEDGMENTS}

We thank Jean-Michel Raimond for insightful discussions about this work. The authors acknowledge funding from the Juan de la Cierva MICINN Program, Spanish projects MICINN FIS2009-10061 and FIS2009-12773C02-01; UPV/EHU UFI 11/55; QUITEMAD; the Basque Government IT472-10; SOLID, CCQED, and PROMISCE European projects; the SFB 631 of the Deutsche Forschungsgemeinschaft; and the German Excellence Initiative via NIM. 
[1] E. T. Jaynes and F.W. Cummings, Comparison of Quantum and Semiclassical Radiation Theories with Application to the Beam Maser, Proc. IEEE 51, 89 (1963).

[2] H. Mabuchi and A.C. Doherty, Cavity Quantum Electrodynamics: Coherence in Context, Science 298, 1372 (2002); A. Ourjoumtsev, A. Kubanek, M. Koch, C. Sames, P.W.H. Pinkse, G. Rempe, and K. Murr, Observation of Squeezed Light from One Atom Excited with Two Photons, Nature (London) 474, 623 (2011).

[3] J.M. Raimond, M. Brune, and S. Haroche, Manipulating Quantum Entanglement with Atoms and Photons in a Cavity, Rev. Mod. Phys. 73, 565 (2001).

[4] R. J. Thompson, G. Rempe, and H. J. Kimble, Observation of Normal-Mode Splitting for an Atom in an Optical Cavity, Phys. Rev. Lett. 68, 1132 (1992); A. Boca, R. Miller, K. M. Birnbaum, A. D. Boozer, J. McKeever, and H. J. Kimble, Observation of the Vacuum Rabi Spectrum for One Trapped Atom, Phys. Rev. Lett. 93, 233603 (2004).

[5] M. Brune, F. Schmidt-Kaler, A. Maali, J. Dreyer, E. Hagley, J. M. Raimond, and S. Haroche, Quantum Rabi Oscillation: A Direct Test of Field Quantization in a Cavity, Phys. Rev. Lett. 76, 1800 (1996).

[6] A. Blais, R.-S. Huang, A. Wallraff, S. M. Girvin, and R. J. Schoelkopf, Cavity Quantum Electrodynamics for Superconducting Electrical Circuits: An Architecture for Quantum Computation, Phys. Rev. A 69, 062320 (2004); A. Wallraff, D. I. Schuster, A. Blais, L. Frunzio, R.-S. Huang, J. Majer, S. Kumar, S. M. Girvin, and R. J. Schoelkopf, Strong Coupling of a Single Photon to a Superconducting Qubit Using Circuit Quantum Electrodynamics, Nature (London) 431, 162 (2004).

[7] J. Clarke and F. K. Wilhelm, Superconducting Quantum Bits, Nature (London) 453, 1031 (2008).

[8] G. Günter, A. A. Anappara, J. Hees, A. Sell, G. Biasiol, L. Sorba, S. De Liberato, C. Ciuti, A. Tredicucci, A. Leitenstorfer, and R. Huber, Sub-Cycle Switch-On of Ultrastrong Light-Matter Interaction, Nature (London) 458, 178 (2009); A. A. Anappara, S. De Liberato, A. Tredicucci, C. Ciuti, G. Biasiol, L. Sorba, and F. Beltram, Signatures of the Ultrastrong Light-Matter Coupling Regime, Phys. Rev. B 79, 201303 (2009).

[9] T. Niemczyk, F. Deppe, H. Huebl, E. P. Menzel, F. Hocke, M. J. Schwarz, J. J. García-Ripoll, D. Zueco, T. Hümmer, E. Solano, A. Marx, and R. Gross, Circuit Quantum Electrodynamics in the Ultrastrong-Coupling Regime, Nature Phys. 6, 772 (2010).

[10] P. Forn-Díaz, J. Lisenfeld, D. Marcos, J. J. García-Ripoll, E. Solano, C.J.P.M. Harmans, and J.E. Mooij, Observation of the Bloch-Siegert Shift in a QubitOscillator System in the Ultrastrong Coupling Regime, Phys. Rev. Lett. 105, 237001 (2010).

[11] C. Ciuti, G. Bastard, and I. Carusotto, Quantum Vacuum Properties of the Intersubband Cavity Polariton Field, Phys. Rev. B 72, 115303 (2005).

[12] J. Bourassa, J. M. Gambetta, A. A. Abdumalikov Jr., O. Astafiev, Y. Nakamura, and A. Blais, Ultrastrong Coupling Regime of Cavity QED with Phase-Biased Flux Qubits, Phys. Rev. A 80, 032109 (2009).
[13] B. Peropadre, P. Forn-Díaz, E. Solano, and J. J. GarcíaRipoll, Switchable Ultrastrong Coupling in Circuit QED, Phys. Rev. Lett. 105, 023601 (2010).

[14] J. Casanova, G. Romero, I. Lizuain, J. J. García-Ripoll, and E. Solano, Deep Strong Coupling Regime of the Jaynes-Cummings Model, Phys. Rev. Lett. 105, 263603 (2010).

[15] I. Buluta and F. Nori, Quantum Simulators, Science 326, 108 (2009).

[16] D. Braak, Integrability of the Rabi Model, Phys. Rev. Lett. 107, 100401 (2011); see also E. Solano, The Dialogue between Quantum Light and Matter, Physics 4, 68 (2011).

[17] J. Larson, Jahn-Teller Systems from a Cavity QED Perspective, Phys. Rev. A 78, 033833 (2008); C.P. Meaney, T. Duty, R. H. McKenzie, and G. J. Milburn, Jahn-Teller Instability in Dissipative Quantum Systems, Phys. Rev. A 81, 043805 (2010).

[18] E. Solano, G. S. Agarwal, and H. Walther, Strong-DrivingAssisted Multipartite Entanglement in Cavity QED, Phys. Rev. Lett. 90, 027903 (2003).

[19] D. Zueco, G. M. Reuther, S. Kohler, and P. Hänggi, QubitOscillator Dynamics in the Dispersive Regime: Analytical Theory Beyond the Rotating-Wave Approximation, Phys. Rev. A 80, 033846 (2009).

[20] S. Deléglise, I. Dotsenko, C. Sayrin, J. Bernu, M. Brune, J. M. Raimond, and S. Haroche, Reconstruction of NonClassical Cavity Field States with Snapshots of Their Decoherence, Nature (London) 455, 510 (2008).

[21] M. Hofheinz, H. Wang, M. Ansmann, R. C. Bialczak, E. Lucero, M. Neeley, A. D. O'Connell, D. Sank, J. Wenner, J. M. Martinis, and A. N. Cleland, Synthesizing Arbitrary Quantum States in a Superconducting Resonator, Nature (London) 459, 546 (2009).

[22] S. Ashhab and F. Nori, Qubit-Oscillator Systems in the Ultrastrong-Coupling Regime and Their Potential for Preparing Nonclassical States, Phys. Rev. A 81, 042311 (2010).

[23] E. P. Menzel, F. Deppe, M. Mariantoni, M. Á. Araque Caballero, A. Baust, T. Niemczyk, E. Hoffmann, A. Marx, E. Solano, and R. Gross, Dual-Path State Reconstruction Scheme for Propagating Quantum Microwaves and Detector Noise Tomography, Phys. Rev. Lett. 105, 100401 (2010); D. Bozyigit, C. Lang, L. Steffen, J. M. Fink, C. Eichler, M. Baur, R. Bianchetti, P. J. Leek, S. Filipp, M. P. da Silva, A. Blais, and A. Wallraff, Antibunching of Microwave-Frequency Photons Observed in Correlation Measurements Using Linear Detectors, Nature Phys. 7, 154 (2010).

[24] O. Astafiev, K. Inomata, A. O. Niskanen, T. Yamamoto, Yu. A. Pashkin, Y. Nakamura, and J.S. Tsai, Single Artificial-Atom Lasing, Nature (London) 449, 588 (2007); M. Grajcar, S. H. W. van der Ploeg, A. Izmalkov, E. Il'ichev, H.-G. Meyer, A. Fedorov, A. Shnirman, and G. Schön, Sisyphus Cooling and Amplification by a Superconducting Qubit, Nature Phys. 4, 612 (2008).

[25] L. Lamata, J. León, T. Schätz, and E. Solano, Dirac Equation and Quantum Relativistic Effects in a Single Trapped Ion, Phys. Rev. Lett. 98, 253005 (2007); R. Gerritsma, G. Kirchmair, F. Zähringer, E. Solano, R. Blatt, and C.F. Roos, Quantum Simulation 
of the Dirac Equation, Nature (London) 463, 68 (2010).

[26] M. Steffen, J.M. Martinis, and I.L. Chuang, Accurate Control of Josephson Phase Qubits, Phys. Rev. B 68, 224518 (2003); F. Motzoi, J. M. Gambetta, P. Rebentrost, and F. K. Wilhelm, Simple Pulses for Elimination of Leakage in Weakly Nonlinear Qubits, Phys. Rev. Lett. 103, 110501
(2009); A. Fedorov, L. Steffen, M. Baur, and A. Wallraff, Implementation of a Toffoli Gate with Superconducting Circuits, Nature (London) 481, 170 (2011).

[27] L. Lamata, J. Casanova, R. Gerritsma, C. F. Roos, J. J. García-Ripoll, and E. Solano, Relativistic Quantum Mechanics with Trapped Ions, New J. Phys. 13, 095003 (2011). 